\title{
Erratum to: Degree of urbanization and gender differences in substance use among Slovak adolescents
}

Lukas Pitel • Andrea Madarasova Geckova •

Jitse P. van Dijk · Sijmen A. Reijneveld

Published online: 1 February 2011

(C) Swiss School of Public Health 2011

Erratum to: Int J Public Health

DOI 10.1007/s00038-010-0219-3

Unfortunately, first names and surnames of the authors were written incorrectly. They should read Lukas Pitel, Andrea Madarasova Geckova, Jitse P. van Dijk, Sijmen A. Reijneveld.

The online version of the original article can be found under doi:10.1007/s00038-010-0219-3

L. Pitel ( $₫)$ · A. Madarasova Geckova · J. P. van Dijk

Medical Faculty, Kosice Institute for Society and Health,

Graduate School and Health Psychology Unit,

Public Health Institute, PJ Safarik University Kosice,

Trieda SNP 1, 04011 Kosice, Slovakia

e-mail: lukas.pitel@upjs.sk

A. Madarasova Geckova

e-mail: geckova@upjs.sk

J. P. van Dijk

e-mail: j.p.van.dijk@med.umcg.nl

J. P. van Dijk · S. A. Reijneveld

Department of Social Medicine, University Medical Center

Groningen, Groningen University, Groningen, The Netherlands

S. A. Reijneveld

e-mail: s.a.reijneveld@med.umcg.nl 